\title{
Dysregulated mRNA Translation in the G2019S LRRK2 and LRRK2 Knock-Out Mouse Brains
}

\author{
(D) Jungwoo Wren Kim, ${ }^{1,2,3}$ Xiling Yin, ${ }^{1,4}$ lan Martin, ${ }^{1,4}$ Yulan Xiong, ${ }^{1,4}$ Stephen M. Eacker, ${ }^{1,4,5}$ \\ Nicholas T. Ingolia, ${ }^{3}$ (-) Ted M. Dawson, ${ }^{1,4,5,6,7,8}$ and ${ }^{-V a l i n a ~ L . ~ D a w s o n ~}{ }^{1,2,4,5,6,8}$
}

\section{https://doi.org/10.1523/ENEURO.0310-21.2021}

\begin{abstract}
${ }^{1}$ Neuroregeneration and Stem Cell Programs, Institute for Cell Engineering, Johns Hopkins University School of Medicine, Baltimore, MD 21205, ²Department of Physiology, Johns Hopkins University School of Medicine, Baltimore, MD 21205, ${ }^{3}$ Department of Molecular and Cell Biology, University of California, Berkeley, Berkeley, CA 94720, ${ }^{4}$ Department of Neurology, Johns Hopkins University School of Medicine, Baltimore, MD 21205, ${ }^{5}$ Adrienne Helis Malvin Medical Research Foundation, New Orleans, LA 70130, ${ }^{6}$ Solomon H. Snyder Department of Neuroscience, Johns Hopkins University School of Medicine, Baltimore, MD 21205, ${ }^{7}$ Department of Pharmacology and Molecular Sciences, Johns Hopkins University School of Medicine, Baltimore, MD 21205, and ${ }^{8}$ Diana Helis Henry Medical Research Foundation, New Orleans, LA 70130
\end{abstract}

\section{Visual Abstract}

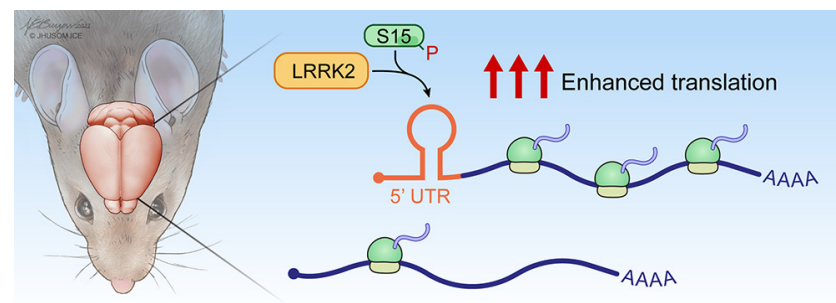

The G2019S mutation in leucine-rich repeat kinase 2 (LRRK2) causes familial Parkinson's disease (PD) and is also found in a subset of idiopathic cases. Prior studies in Drosophila and human induced pluripotent stem cell (iPSC)-derived dopamine neurons uncovered a pronounced effect of G2019S LRRK2 on mRNA translation. It was previously reported that G2019S LRRK2 promotes translation of mRNAs with complex 5' untranslated region (UTR) secondary structure, resulting in increased expression of calcium channels and dysregulated calcium homeostasis in human dopamine neurons. Here, we show that dysregulated translation occurs in the brains of mammalian LRRK2 models in vivo. Through ribosome profiling studies of global translation, we observe that mRNAs with complex 5'UTR structure are also preferentially translated in the G2019S LRRK2-expressing mouse brain. Reporter assays suggest that this $5^{\prime}$ UTR preference is independent of translation initiation factors. Conversely, translation of mRNAs with complex 5'UTR secondary structure is downregulated

\section{Significance Statement}

Parkinson's disease (PD)-linked G2019S mutation of leucine-rich repeat kinase 2 (LRRK2) is known to cause abnormalities in mRNA translation. These translational defects were suggested to cause calcium dysregulation, thereby imposing a long-term cellular stress to dopamine neurons. While these effects of G2019S LRRK2 on mRNA translation have been seen in Drosophila brain tissues and cultured mammalian neurons, translational profiling of the mammalian brain expressing G2019S LRRK2 has not been reported. In this study, we employed ribosome profiling to survey mRNA translation in the brains of LRRK2 mouse models, thereby demonstrating that the G2019S LRRK2 mutation broadly alters mRNA translation in the mouse brain. 
in LRRK2 knock-out (KO) mouse brain, indicating a robust link between LRRK2 kinase activity and translation of mRNA with complex 5'UTR structure. Further, substantia nigra pars compacta (SNpc) dopamine neurons in the G2019S LRRK2-expressing brain exhibit increased calcium influx, which is consistent with the previous report from human dopamine neurons. These results collectively suggest that LRRK2 plays a mechanistic role in translational regulation, and the G2019S mutation in LRRK2 causes translational defects leading to calcium dysregulation in the mammalian brain.

Key words: LRRK2; RPS15; translation

\section{Introduction}

Dominant mutations in the leucine-rich repeat kinase 2 (LRRK2) gene are the most common genetic cause of familial Parkinson's disease (PD), with the G2019S missense mutation being most frequent disease-causing mutation in LRRK2 (Martin et al., 2014a). The G2019S mutation enhances the kinase activity of LRRK2, leading to neurotoxicity (Greggio et al., 2006; Smith et al., 2006). While various cellular functions are associated with LRRK2 kinase activity, emerging evidence suggests that alterations in mRNA translation downstream of kinase activity plays an important role in PD pathogenesis (Imai et al., 2008; Gehrke et al., 2010; Martin et al., 2014b; Taymans et al., 2015). G2019S LRRK2 was reported to increase global protein synthesis through phosphorylation of the ribosomal protein S15 (uS19), and reduction of global protein synthesis is protective against G2019S LRRK2 neurotoxicity in a Drosophila model (Martin et al., 2014b). In addition, a recent study applying ribosome profiling to human dopamine neurons differentiated from

Received July 20, 2021; accepted October 28, 2021; First published November 10, 2021.

The authors declare no competing financial interests.

Author contributions: J.W.K., T.M.D., and V.L.D. designed research; J.W.K., X.Y., I.M., Y.X., and N.T.I. performed research; J.W.K. and S.M.E. contributed unpublished reagents/analytic tools; J.W.K., X.Y., I.M., and N.T.I. analyzed data; J.W.K., T.M.D., and V.L.D. wrote the paper.

This work was supported by the National Institutes of Health (NIH) Grant P50 NS38377, the JPB Foundation, and the Adrienne Helis Malvin Medical Research Foundation's Parkinson's Disease Program M-2014. J.W.K was supported by Korea Foundation of Advanced Studies. I.M. was supported by the NIH/National Institute on Aging (NIA) Grant K01-01AG050718. Y.X. was supported by NIH/NIA Grant K01-AG046366 and The William N. \& Bernice E. Bumpus Foundation Innovation Awards.

I. Martin's present address: Jungers Center for Neurosciences Research, Parkinson Center of Oregon, Department of Neurology, Oregon Health and Science University, Portland, OR 97239.

Y. Xiong's present address: Department of Neuroscience, University of Connecticut School of Medicine, Farmington, CT 06030.

S. M. Eacker's present address: Phase Genomics Inc, Seattle, WA 98109.

Acknowledgements: We thank the joint participation by the Adrienne Helis Malvin Medical Research Foundation through its direct engagement in the continuous active conduct of medical research in conjunction with The Johns Hopkins Hospital and the Johns Hopkins University School of Medicine. The IRES reporters are gifts from P. A. Sharp's laboratory (Addgene \#11509, \#11510). T.M.D. is the Leonard and Madlyn Abramson Professor in Neurodegenerative Diseases.

Correspondence should be addressed to Valina L. Dawson at vdawson@ jhmi.edu or Ted M. Dawson at tdawson@jhmi.edu.

https://doi.org/10.1523/ENEURO.0310-21.2021 Copyright @ $2021 \mathrm{Kim}$ et al.

This is an open-access article distributed under the terms of the Creative Commons Attribution 4.0 International license, which permits unrestricted use, distribution and reproduction in any medium provided that the original work is properly attributed. patient-derived induced pluripotent stem cells (iPSCs) showed that the increased translation in G2019S LRRK2 leads to increased expression of genes responsible for calcium influx in neurons (Kim et al., 2020). While these studies presented potential mechanisms linking abnormal translation to cellular stress, the proposed mechanisms have yet to be tested in the mammalian brain.

\section{Materials and Methods}

All animal protocols are in accordance with the regulations of Johns Hopkins University Animal Care and Use Committee and the National Institutes of Health Guide for the Care and Use of Laboratory Animals. Animals were housed in a 12/12 $\mathrm{h}$ light/dark cycle with free access to water and food. High-throughput sequencing data are available via NCBI GEO (accession number: GSE167704).

\section{Maintenance of LRRK2 transgenic mouse models}

Generation and characterization of LRRK2 "Tet-off" transgenic mice and LRRK2 knock-out (KO) mice were previously reported (Andres-Mateos et al., 2009; Nikonova et al., 2012; Xiong et al., 2017). For transgenic mice, high copy number lines (569 line for GS, 763 line for GS/DA) were used (Xiong et al., 2017). Single transgenic mice $\mathrm{Ca}^{2+} /$ calmodulin-dependent protein kinase II (CaMKII)-tTA or Tet-LRRK2) were used for breeding, and the breeding cages were maintained with doxycycline chow (Diet-Sterile, $200 \mathrm{mg} / \mathrm{kg}$ doxycycline, BioServ) and fed ad libitum. Doxycycline food was switched back to regular food after weaning for transgene induction. Three- to four-month-old mice were used for ribosome profiling experiments (described below).

\section{Mouse primary cortical neuron culture}

Dissipated primary cortical neurons were prepared from embryonic day (E)15 developing brain (CD1, Charles River or LRRK2 transgenic mice). Developing cortices were dissected in the dissecting medium (DMEM with $20 \%$ horse serum, $0.5 \mathrm{~mm}$ GlutaMax, and $6 \mu \mathrm{m}$ glucose, Invitrogen), digested with TrypLE (Invitrogen), and plated at a concentration of $3 \times 10^{6}$ cells for a plate. Culture plates were precoated with $15 \mu \mathrm{g} / \mathrm{ml}$ poly-L-ornithine. Cultures were maintained under Neurobasal (Invitrogen) medium with a serum-free supplement B-27 (Invitrogen) and $0.5 \mathrm{~mm}$ GlutaMax (Invitrogen).

\section{Immunocytochemistry of neurons}

Cells were fixed with $4 \%$ paraformaldehyde for $15 \mathrm{~min}$ at room temperature, then permeabilized with $0.03 \%$ Triton X-100 for $15 \mathrm{~min}$. The cells were washed then blocked for $1 \mathrm{~h}$ with $10 \%$ goat serum in PBS. The blocked 
cells were subsequently incubated with primary antibody for overnight at $4{ }^{\circ} \mathrm{C}$. On the following day, the cells were incubated with secondary antibody for $1 \mathrm{~h}$ at room temperature in a light controlled condition. After $3 \times$ wash with PBS buffer, the cells were mounted on cover slides with mounting media containing DAPI. All images were taken for analysis with Zeiss AxioObserver Z1 or LSM710 (Carl Zeiss) confocal laser scanning microscope under $20 \times$ or $40 \times$ oil objectives. Blinding was not performed with immunocytochemistry experiments. The following primary antibodies were used for immunocytochemistry: $\alpha$-tyrosine hydroxylase (TH; 1:1000, EMD Millipore AB152).

\section{Ribosome profiling library generation}

Ribosome footprinting and RNA-seq libraries were prepared by following a published protocol with several modifications made for mouse brain tissue (Ingolia et al., 2012).

\section{Mouse brain}

Brains of three- to four-month-old mice were dissected in TBS buffer with $100 \mu \mathrm{g} / \mathrm{ml}$ cycloheximide and immediately frozen in dry ice. Caudate putamen tissues from three mice of mixed gender (1:2 or 2:1 male:female ratio) were pooled; $2.5 \%$ of total lysate was subjected to Western blotting to ensure sufficient expression of transgene. The collected samples were homogenized in lysis buffer [10 mм Tris pH 7.5, $150 \mathrm{~mm} \mathrm{NaCl,} 5 \mathrm{~mm} \mathrm{MgCl}$, 0.5 $\mathrm{mm}$ DTT, $100 \mu \mathrm{g} / \mathrm{ml}$ cycloheximide, EDTA-free protease inhibitor (Roche), and $40 \mathrm{U} / \mathrm{ml}$ murine RNase Inhibitor (NEB)] with 12 strokes of high-speed motorized homogenizer (Glas-Col GT series) at $40 \%$ power. The lysates were briefly centrifuged for $10 \mathrm{~min}$ at $2000 \times \mathrm{g}$. The supernatant was transferred to a new tube, added NP-40-1\% final concentration, incubated $5 \mathrm{~min}$ on ice. The samples were centrifuged again for $10 \mathrm{~min}$ at $20,000 \times \mathrm{g}$. The lysates were incubated in ice for $15 \mathrm{~min}$, and centrifuged for $10 \mathrm{~min}$ at $20,000 \times g$. Total RNA concentration of lysate was measured by Qubit RNA BR Assay (Life Technologies), and the same amount of RNA was used across samples. The supernatant was split into two tubes for ribosome footprinting and RNAseq library generation.

\section{Ribosome footprinting}

The lysates were treated with $15 \mu \mathrm{l}$ of RNase I (Ambion) in $600-\mu$ l total reaction volume for $45 \mathrm{~min}$ at room temperature, and the reaction was stopped by adding $30 \mu \mathrm{l}$ of SuperAse-In (Ambion). Sucrose cushion was performed with $1.7 \mathrm{~g}$ sucrose in $3.9 \mathrm{ml}$ polysome buffer $(10 \mathrm{~mm}$ Tris $\mathrm{pH} 7.5$, $150 \mathrm{~mm} \mathrm{NaCl}, 5 \mathrm{~mm} \mathrm{MgCl} 2,0.5 \mathrm{~mm}$ DTT, $100 \mu \mathrm{g} / \mathrm{ml}$ cycloheximide, 20U/ml SuperAse-In), $4 \mathrm{~h}$ at 70,000rpm. The pellet was resuspended with $700 \mu \mathrm{l}$ QIAzol (QIAGEN) reagent, incubated for $5 \mathrm{~min}$ at room temperature, $140 \mu \mathrm{l}$ chloroform was added, vortexed for $15 \mathrm{~s}$, and incubated again for $2 \mathrm{~min}$ at room temperature. The sample was centrifuged for $15 \mathrm{~min}$ at $12,000 \times$ $g$, the $350 \mu \mathrm{l}$ supernatant was mixed with $525 \mu \mathrm{l} 100 \% \mathrm{EtOH}$. The mixture was loaded on an RNeasy Mini column (QIAGEN), and the RNA was extracted; 26-34 nt ribosome footprints were size-selected by Urea-PAGE, gel extraction and RNA purification. Ribo-Zero Gold kit (lllumina) was used for rRNA removal after the size selection. The rRNA depleted ribosome footprints were dephosphorylated by T4 polynucleotide kinase treatment, then Universal miRNA Cloning Linker (NEB) was added to the $3^{\prime}$ ends. Reverse transcription reaction was performed, and the cDNA was circularized by CircLigase II (Epicentre) reaction, and subjected to the PCR for final library generation.

\section{RNA-seq}

Total RNA was purified by a combination of QIAzol and RNeasy Mini as described. Ribo-Zero Gold kit was used for rRNA removal. RNA-seq library was generated from the total RNA by ScriptSeq v2 Library Preparation kit (Epicentre).

\section{Ribosome profiling data processing}

Illumina HiSeq 2000 or 2500 were used for deep sequencing of the libraries. FASTX-Toolkit (http://hannonlab.cshl.edu/ fastx_toolkit/) was used for the initial processing of the reads.

\section{Ribosome footprinting libraries}

Only adapter-containing reads were clipped. Reads shorter than $25 \mathrm{nt}$ were discarded. The first nucleotide of the reads was trimmed. rRNA-mapped reads were discarded before genomic alignment.

\section{RNA-seq libraries}

Only adapter-containing reads were clipped, rRNAmapped reads were discarded.

The processed reads were mapped to the UCSC genome database (mouse: $\mathrm{mm} 9$ ) by Tophat (2.0.11) with Bowtie2 (2.2.2). Maximum 1 mismatch was allowed for the alignments (for sequencing read counts, see Table 1).

\section{Internal ribosome entry site (IRES) reporter assays}

pFR-HCV-xb, pFR-CrPV-xb vectors (from Phil Sharp Lab) were obtained from the Addgene depository (\#11510 and \#11509, respectively). The reporter vectors were cotransfected into CD1 wild-type mouse cortical neurons at day in vitro (DIV)5 with LRRK2-expressing or S15-expressing plasmids (or empty, respective expression plasmids for control) using Lipofectamine 2000 (Invitrogen) reagent. Luciferase to LRRK2/S15 expression vector ratio was 1:3. Culture medium was replaced (half-change) every $24 \mathrm{~h}$ to minimize any potential effects from the growth condition including starvation. Luciferase activity was measured at DIV7 by Dual-Glo Luciferase Assay System (Promega; for the IRES reporters) with Glomax 20/20 Luminometer (Promega). The lysates were subjected to the total RNA purification with DNase treatment for the transcript level measurement.

\section{Immunoblotting}

Brain tissues were lysed with an automated homogenizer in RIPA buffer with 1\% SDS $[20 \mathrm{~mm}$ Tris- $\mathrm{HCl}(\mathrm{pH}$ 7.5), $150 \mathrm{~mm} \mathrm{NaCl}, 1 \mathrm{~mm}$ EDTA, 1\% NP-40, 1\% sodium deoxycholate, $1 \%$ SDS, protease inhibitors]. Lysates were incubated on a rotator for $1 \mathrm{~h}$ at $4^{\circ} \mathrm{C}$, and spun down for $10 \mathrm{~min} \times 12,000 \times g$ at $4^{\circ} \mathrm{C}$. Supernatant was collected, protein concentration was measured, and the lysate was mixed with $2 \times$ Laemmli sample buffer. Generation and 
Table 1. Sequencing read counts

\begin{tabular}{|c|c|c|}
\hline Sample & Type & Mapped reads \\
\hline Mouse control 1 & Ribo & $11,528,964$ \\
\hline mRNA & $35,167,826$ & \\
\hline Mouse control 2 & Ribo & $14,236,436$ \\
\hline mRNA & $53,086,785$ & \\
\hline Mouse control 3 & Ribo & $28,913,143$ \\
\hline mRNA & $37,446,599$ & \\
\hline G2019S TG 1 & Ribo & $10,832,574$ \\
\hline mRNA & $37,329,310$ & \\
\hline G2019S TG 2 & Ribo & $11,636,313$ \\
\hline mRNA & $42,813,645$ & \\
\hline G2019S/D1994A TG 1 & Ribo & $11,391,779$ \\
\hline mRNA & $62,883,025$ & \\
\hline G2019S/D1994A TG 2 & Ribo & $30,967,177$ \\
\hline mRNA & $33,887,256$ & \\
\hline Mouse WT (vs KO) 1 & Ribo & $6,069,632$ \\
\hline mRNA & $44,668,380$ & \\
\hline $\begin{array}{l}\text { Mouse WT (vs KO) } 2 \\
\text { mRNA }\end{array}$ & $\begin{array}{l}\text { Ribo } \\
8,025,070\end{array}$ & $6,331,204$ \\
\hline LRRK2 KO 1 & Ribo & $5,552,451$ \\
\hline mRNA & $65,571,861$ & \\
\hline LRRK2 KO 2 & Ribo & $7,322,616$ \\
\hline mRNA & $18,455,674$ & \\
\hline LRRK2 WT 3 (STR) & Ribo & $22,256,190$ \\
\hline LRRK2 WT 3 (VMB) & Ribo & $8,773,494$ \\
\hline LRRK2 KO 3 (STR) & Ribo & $22,069,910$ \\
\hline LRRK2 KO 3 (VMB) & Ribo & $6,492,191$ \\
\hline
\end{tabular}

Ribo: ribosome profiling; mRNA: RNA-Seq; TG: transgenic mice; WT: wild type, KO: knock-out mice; STR: striatum; VMB: ventral midbrain.

characterization of rabbit polyclonal T136 phospho-S15 antibody was previously published (Martin et al., 2014b). Commercial antibodies: LRRK2: Neuromab (75-188, N138/6), P-elF2a (Cell Signaling Technologies, \#9721), elF2a (Cell Signaling Technologies, \#9722), and ATF4 (Millipore, ABE387).

\section{Ribosome profiling data analysis}

Aligned reads were counted by either a Python package HTSeq (htseq-count) or an R package GenomicAlignments (summerizeOverlaps). Annotations and sequencing reads were handled using an $\mathrm{R}$ package GenomicFeatures. To avoid multiple counting on isoforms, transcript reference data were processed to have one unique annotation covering all isoforms (union of isoforms) per gene. Reads only in the CDS regions were counted. Transcripts with low read counts (<128 reads) were discarded. An $\mathrm{R}$ package DESeq (1.20) was used for calculating normalized expression from either ribosome footprinting or RNA-seq data based on a negative binomial distribution and generalized linear model. For the mouse data, replicates were initially analyzed independently to confirm reproducibility, and then analyzed in combination for the final analysis. For the human neuron data, biological triplicates were handled by DESeq. Translation efficiency (TE) was calculated based on the DESeq expression output. 5' Untranslated region (UTR) estimated folding energy table was extracted from the UCSC genome database (fold5UTR field: mm9). For the 5'UTR estimated folding energy comparison, a control group with similar group size was randomly selected for each comparison to avoid potential bias from sample size differences. Transcript coordinates were calculated by a custom $R$ script and re-aligned based on the rounded half point of the ribosome footprint [5' end + (footprint length/2)]. For icSHAPE data analysis, icSHAPE (in vivo) results from mouse ES cells (GEO: GSE64169) were downloaded, converted to $\mathrm{mm} 9$ (UCSC liftOver), and merged with our mouse ribosome profiling data.

\section{Electrophysiological recordings}

Mice (10-12 weeks old) were anesthetized and decapitated, and the brains were placed in ice-cold artificial CSF (ACSF) containing the following: $125 \mathrm{~mm} \mathrm{NaCl}, 2.5 \mathrm{~mm} \mathrm{KCl}$, $1 \mathrm{~mm} \mathrm{MgSO}_{4}, 1.25 \mathrm{~mm} \mathrm{NaH}_{2} \mathrm{PO}_{4}, 26 \mathrm{~mm} \mathrm{NaHCO}_{3}, 2 \mathrm{~mm}$ $\mathrm{CaCl}_{2}$, and $10 \mathrm{~mm}$ D-glucose. Transverse brain slices containing substantia nigra pars compacta (SNpc; $350 \mu \mathrm{m}$ ) were prepared using a vibratome (Leica VT1200S). Sections were incubated in ACSF saturated with $95 \% \mathrm{O}_{2}$ and $5 \%$ $\mathrm{CO}_{2}$, at $34^{\circ} \mathrm{C}$ for $60 \mathrm{~min}$, and then at room temperature (22$24^{\circ} \mathrm{C}$ ) until use. Recordings were performed at room temperature. All experiments were conducted in accordance with the National Institutes of Health Guide for the Care and Use of Laboratory Animals.

HEKA EPC10 amplifier (HEKA Elektronik) was used to perform electrophysiological recordings. For spontaneous and evoked action potentials (APs), a single slice was transferred into a submerged recording chamber and perfused constantly with oxygenated ACSF at a rate of $2 \mathrm{ml} /$ min. DA neurons were visualized under a $40 \times$ water immersion objective by fluorescence and DIC optics (Carl Zeiss). The patch electrodes had a resistance of 2-5 $\mathrm{M} \Omega$ and filled with solution containing the following: $126 \mathrm{~mm} \mathrm{~K}$ gluconate, $8 \mathrm{~mm} \mathrm{KCl,} 20 \mathrm{~mm}$ HEPES, $0.2 \mathrm{~mm}$ EGTA, $2 \mathrm{~mm}$ $\mathrm{NaCl}, 3 \mathrm{~mm}$ MgATP, $0.5 \mathrm{~mm} \mathrm{Na} \mathrm{N}_{3} \mathrm{GTP}$, and $0.05 \mathrm{~mm}$ Alexa Fluor 568 (pH 7.2, 290-300 mOsm/kg). Input resistance (Rin), series resistance (Rseries), and leak currents (Leak) were monitored throughout the experiment. Unstable recordings ( $>10 \%$ fluctuation of Rseries value) during the course of experiments were rejected for further analysis. Resting membrane potential was recorded in current clamp mode at $0 \mathrm{pA}$ immediately after establishing whole-cell configuration. A series of hyperpolarizing and depolarizing step currents were injected to elicit APs. For wholecell calcium currents, the external solution used contained $140 \mathrm{~mm}$ tetraethylammonium methanesulfonate $\left(\mathrm{TEA}-\mathrm{MeSO}_{3}\right.$ ), $10 \mathrm{~mm}$ HEPES, and $10 \mathrm{~mm} \mathrm{BaCl} 2$ or $\mathrm{CaCl}_{2}(\mathrm{pH} 7.4,300-310 \mathrm{mOsmol} / \mathrm{kg})$. The pipette solution contained $135 \mathrm{~mm} \mathrm{CsMeSO}, 5 \mathrm{~mm} \mathrm{CsCl}, 1 \mathrm{~mm}$ $\mathrm{MgCl}_{2}, 4 \mathrm{~mm}$ MgATP, $5 \mathrm{~mm}$ HEPES, and $5 \mathrm{~mm}$ EGTA (pH 7.3, 290-300 mOsmol/kg). Currents were recorded by holding the cell at $-90 \mathrm{mV}$, before stepping to various potentials from -60 to $+50 \mathrm{mV}$ for 250 -ms in 10 $\mathrm{mV}$ increments. Tetrodotoxin (TTX; $1 \mu \mathrm{M}$ ) was used to block voltage-gated sodium currents. Data were acquired by PatchMaster software (HEKA Elektronik), sampled at $10 \mathrm{kHz}$, and filtered at $2.9 \mathrm{kHz}$. APs and calcium currents were analyzed using Clampfit 10.5 software (Molecular Devices). Neurons labeled with 
Alexa Fluor 568 were confirmed by immunohistochemistry after recording.

\section{Results}

We sought to characterize translational abnormalities in the brains of LRRK2 mouse models, focusing on the caudate putamen, where substantia nigra dopamine neurons project to and is linked to the pathology of PD. To obtain high expression of G2019S LRRK2 or kinase dead G2019S/D1994A LRRK2 transgenes, we crossed mice harboring doxycycline-regulated LRRK2 expression constructs with the CaMKII-tTA driver mice (Lee et al., 2013; Xiong et al., 2017). We then analyzed translation in the caudate putamen of the resulting G2019S LRRK2 or G2019S/D1994A LRRK2 transgenic mice, as well as LRRK2 $\mathrm{KO}$ animals (Fig. 1A; Extended Data Figs. 1-1, 1-2; Nikonova et al., 2012).

We characterized translation by ribosome profiling, the deep sequencing of ribosome-protected mRNA fragments generated by nuclease digestion. Ribosome profiling provides a quantitative measurement of translation and reports on the precise location of translating ribosomes across the transcriptome (Ingolia et al., 2009). We inferred the translational activity of different mRNAs by calculating the TE, the ratio between the abundance of ribosome footprints derived from a gene to the overall abundance of its mRNA as determined by RNA-seq (Anders and Huber, 2010; Brar and Weissman, 2015; Ingolia, 2016). Comparison of the global distribution of TE values between LRRK2 transgenic mice and non-transgenic littermate control mice revealed broad alterations in TE distribution (Fig. 1B). Likewise, LRRK2 KO mice showed widespread differences in TE relative to wild-type control mice (Fig. 1C). In contrast, G2019S/D1994A LRRK2 transgenic mice have a TE distribution similar to those in non-transgenic control mice, indicating that the changes in LRRK2 transgenic mice are dependent on kinase activity (Fig. 1D; Greggio et al., 2006; Smith et al., 2006). The broadly altered TE distribution indicates that G2019S LRRK2 causes increased expression of some genes (TE up) and decreased expression of others (TE down), distorting the overall translatome.

It has been shown that G2019S LRRK2 enhances the translation of transcripts containing complex $5^{\prime}$ UTR structure (Kim et al., 2020). Therefore, we compared the predicted 5'UTR folding energy between genes showing elevated or reduced TE from each comparison. The TE up genes in G2019S LRRK2 transgenic mouse brain have significantly lower folding energy than randomly selected control genes with the same group size (Fig. 2A), indicating that they have more complex 5'UTR secondary structures. Conversely, the TE down genes have significantly higher folding energy compared with the control genes, which suggests that they have less structured 5'UTR (Fig. 2A). Notably, LRRK2 KO mice show the reverse trend, indicating that loss of LRRK2 has the opposite effect from hyperactive G2019S LRRK2 (Fig. 2B). The same trend is clear when we stratify transcripts according to the strength of their $5^{\prime}$ UTR secondary structure (Fig. 2C,D). Unlike the case for $5^{\prime}$ UTRs, 3'UTR folding energy does not show LRRK2-dependent correlation with TE (Extended Data Fig. 2-1A,B). In addition, we did not find significant TE changes from $5^{\prime}$ terminal oligopyrimidine (TOP)-containing genes, which are known to be regulated by phosphorylation of 4E-BP (Extended Data Fig. 2-1C,D; Thoreen et al., 2012). Therefore, our ribosome profiling data from the mouse brain samples indicate that LRRK2 enhances translation of mRNAs with complex 5'UTR secondary structure in a kinase activity-dependent manner.

Recent advances in molecular techniques that combine chemical probes and deep sequencing have provided transcriptome-wide measurements of RNA structure in living cells. We analyzed mouse RNA structure data (icSHAPE; Spitale et al., 2015) to estimate basal levels of 5'UTR structural complexity of genes differentially regulated by LRRK2. Low icSHAPE signal indicates low chemical reactivity at a given nucleotide, thereby suggesting a higher likelihood that it participates in secondary structures in cells. We compared icSHAPE reactivity between TE up and TE down genes from G2019S LRRK2 transgenic and LRRK2 KO mice. Structure probing data from $100 \mathrm{nt}$ windows $5^{\prime}$ of the CDS start site revealed that the TE up genes in G2019S LRRK2 have significantly low average icSHAPE reactivity $(0.229)$ associated with more complex structure, while the TE down genes have higher average reactivity $(0.240)$ suggesting low structural complexity (Fig. 2E). LRRK2 KO mice show the opposite trend (up: 0.237, down: 0.219; Fig. 2F). These results suggest that the $5^{\prime}$ UTR secondary structure adjacent to the start codon may play a role in the translatome alteration by G2019S LRRK2.

Translation initiation is a tightly regulated process, with many eukaryotic initiation factors (elFs) involved in the regulation and facilitation of the process (Sonenberg and Hinnebusch, 2009; Jackson et al., 2010). Of note, DEADbox RNA helicases including elF4A, Ddx3, and Dhx29 are thought to resolve $5^{\prime}$ UTR secondary structure of mRNAs with the help of other initiation factors such as elF4B (Parsyan et al., 2011; Sen et al., 2015). Previous studies suggested that T136 phosphorylation of ribosomal protein S15 (uS19) mediates the translational effects of G2019S LRRK2 (Martin et al., 2014b; Kim et al., 2020). Consistent with this, we found that S15 T136 phosphorylation is increased in the G2019S LRRK2 transgenic mouse brain and decreased in the LRRK2 KO mouse brain (Fig. $3 A, B$ ). To investigate potential crosstalk between G2019S LRRK2, phosphorylated S15 and elFs, we employed bicistronic reporters with hepatitis $C$ virus (HCV) or cricket paralysis virus (CrPV) IRES. HCV-IRES and CrPV-IRES do not require RNA helicase activity to initiate translation, and CrPV-IRES initiation is entirely independent of elFs (Fig. 3C,D; Jackson et al., 2010). In these bicistronic reporter assays, cap-dependent translation of firefly luciferase is dependent on helicase activity of elFs, while IRES-driven cap-independent translation of Renilla luciferase is helicase independent. Unexpectedly, both IRES reporters show the same cap-dependent and cap-independent translational induction by G2019S LRRK2 and T136D S15, 
A Mouse brain tissue

- GS LRRK2

- GS/DA LRRK2

- LRRK2 KO
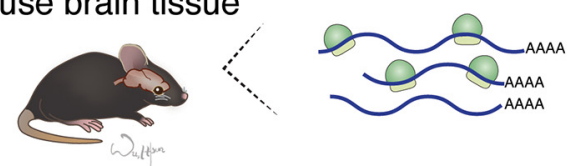

Ribosome footprinting library

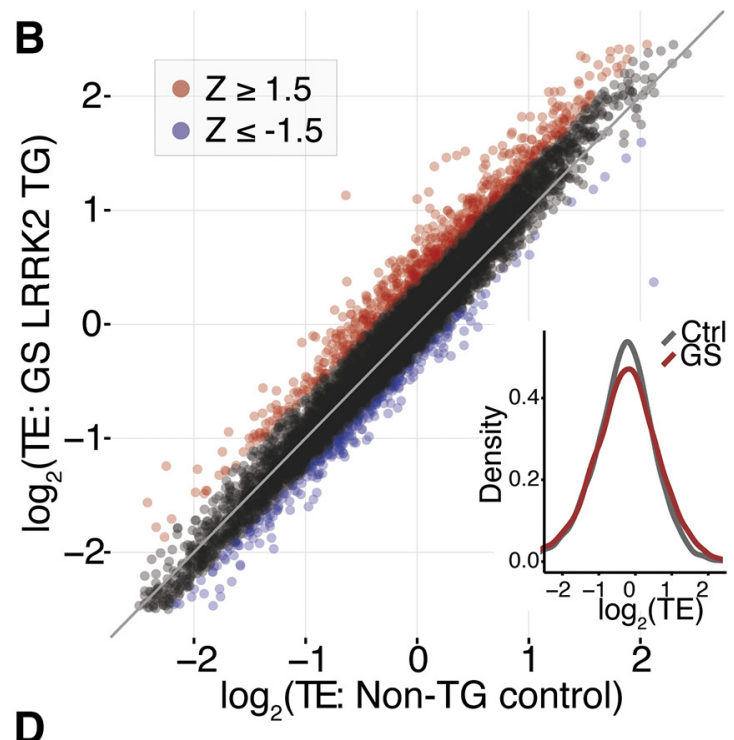

C 2-
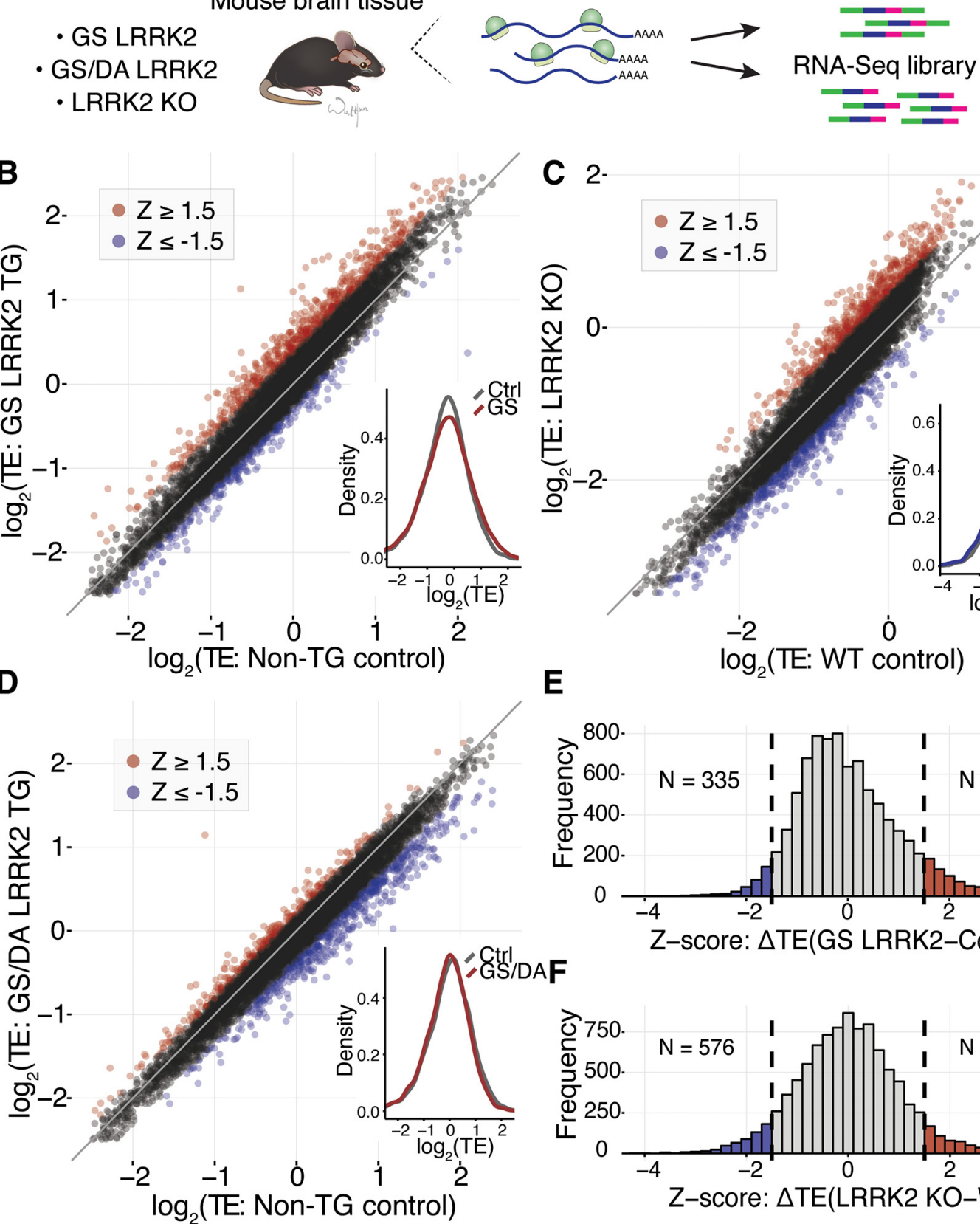

E
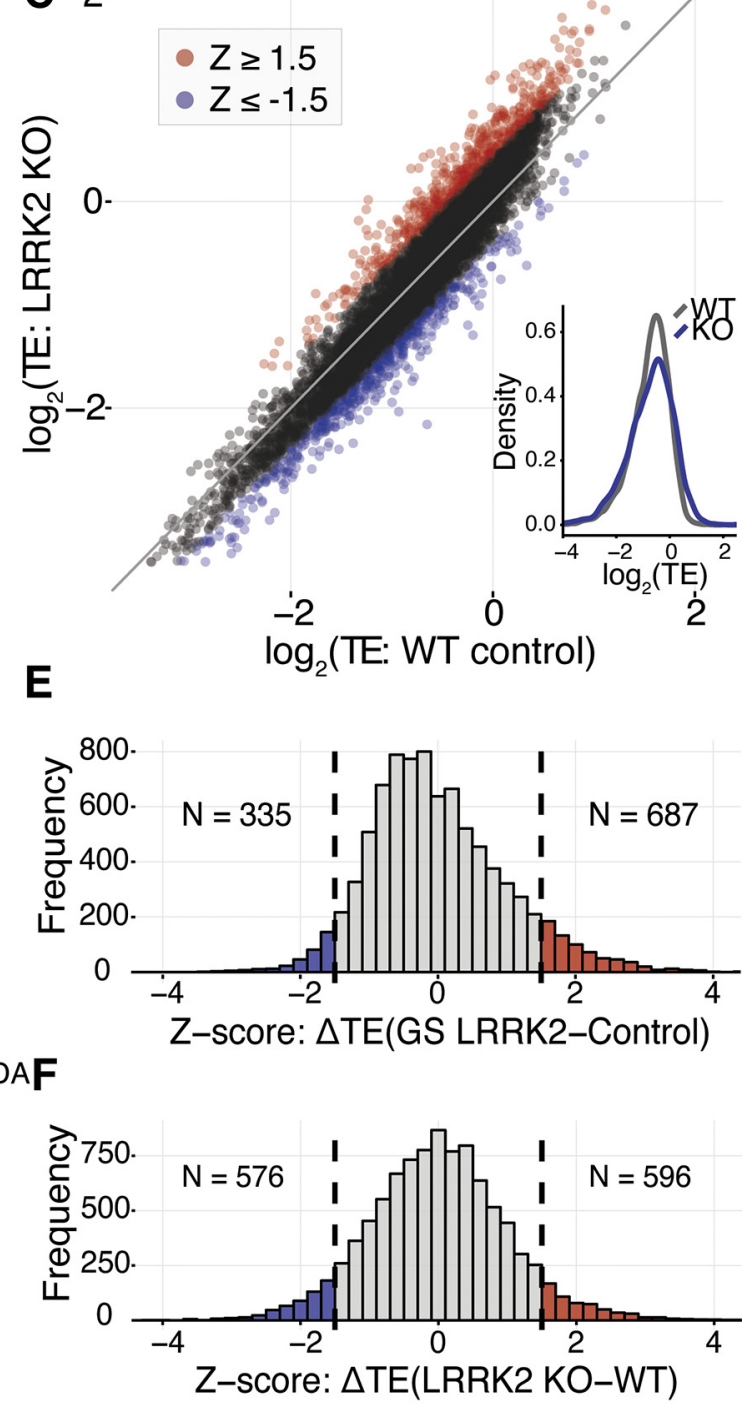

Figure 1. Broad alteration in mRNA translation in the G2019S LRRK2 mouse brain. $\boldsymbol{A}$, A schematic of ribosome profiling workflow with mouse brain tissue. $\boldsymbol{B}-\boldsymbol{D}$, TE was calculated to estimate translational activity. Global TE distributions between (B) GS LRRK2 TG and non-TG control, (C) LRRK2 KO and WT, and (D) GS/DA LRRK2 TG and non-TG control were compared. All values are in $\log _{2}$, and each data point represents a single transcript. In scatterplots, centerline is a guideline with slope of 1, meaning that the dots on the line do not have TE value differences between the genotypes. SD of TE differences: 0.226 (GS LRRK2 vs control), 0.179 (GS/DA LRRK2 vs control), 0.273 (LRRK2 KO vs WT). Standard $z$ score was calculated, and \pm 1.5 cutoff was used to select TE up and TE down genes. Triplet periodicity is normal across the results (Extended Data Fig. 1-1). $\boldsymbol{E}$, $\boldsymbol{F}$, Histogram of TE differences $(\delta$ TE, $\triangle T E$ ) between $(E)$ GS LRRK2 TG and non-TG control or $(\boldsymbol{F})$ LRRK2 KO and WT. $Z$ score \pm 1.5 cutoff was used, and TE values are in $\log _{2}$. Each ribosome profiling experiment was firstly analyzed independently to ensure reproducibility. Two independent results were analyzed together by DESeq (Anders and Huber, 2010; $n=2$ ). Expression analysis results including TE values were compiled (Extended Data Fig. 1-2).

thereby leaving the ratios between cap-dependent and cap-independent translation unchanged (Fig. 3E-J; Extended Data Fig. 3-1). Since the CrPV IRES does not require any initiation factors to recruit ribosomes, the results indicate that the translational effects of G2019S LRRK2 are independent of translation initiation factors, and phosphorylation of S15 is sufficient to enhance the translation of mRNAs with structured 5'UTRs. 
A

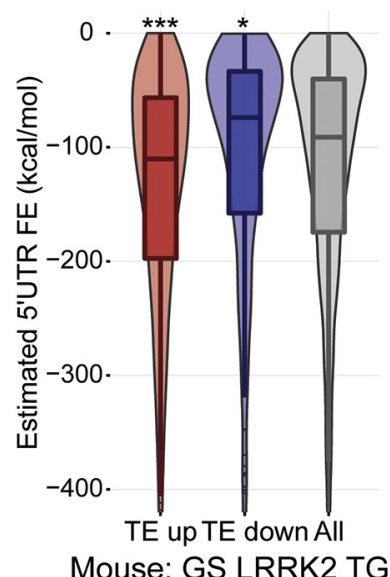

B

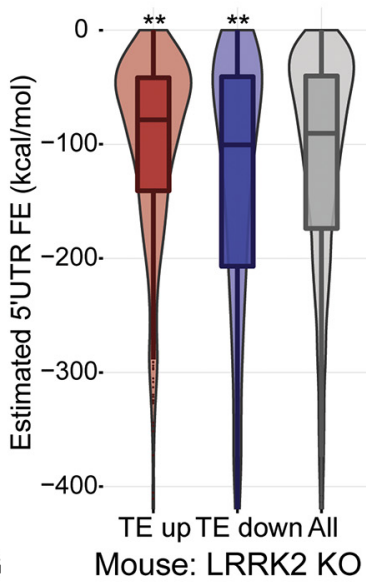

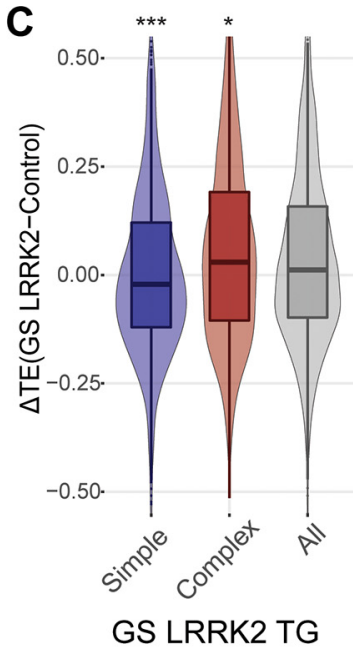

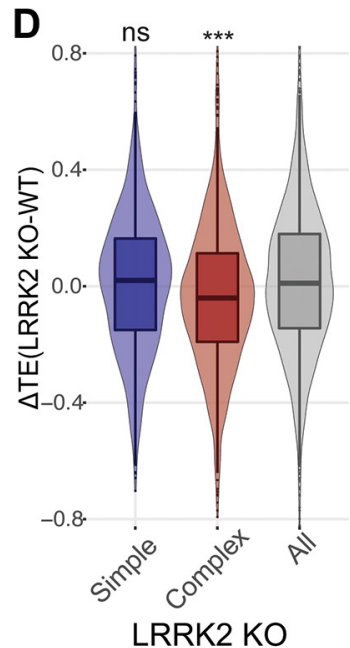

E

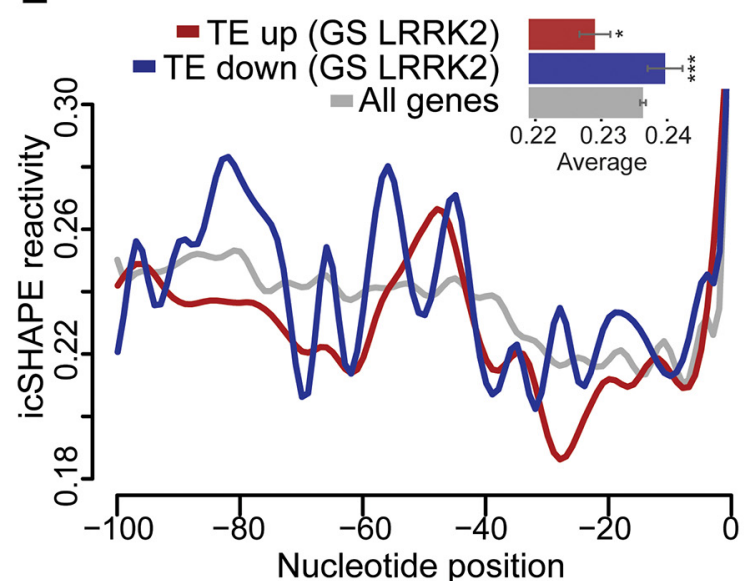

$\mathbf{F}$

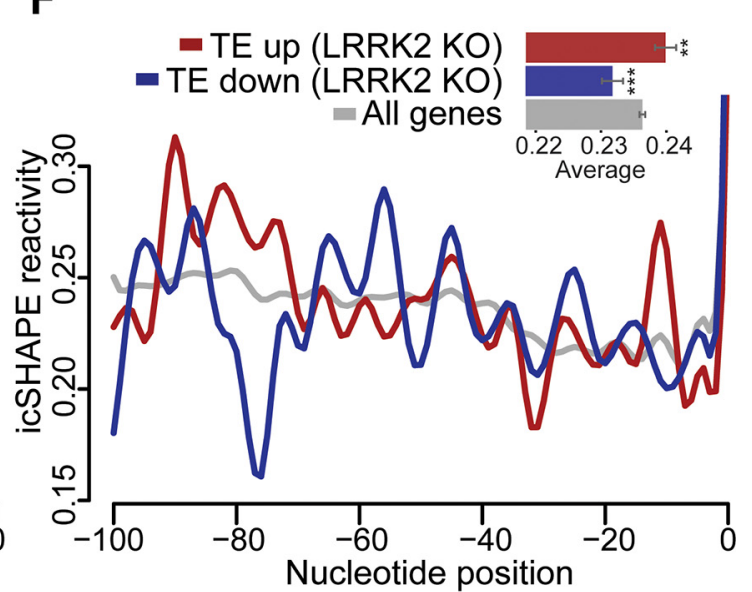

Figure 2. 5'UTR secondary structure mediates translational effects of G2019S LRRK2. $\boldsymbol{A}$, $\boldsymbol{B}$, Correlation between estimated 5'UTR folding energy and TE changes in (A) GS LRRK2 TG or (B) LRRK2 KO. Box plot overlaid with violin plot visualizes the median, the first and the third quartile along with the data distribution pattern. 5'UTR folding energy for transcripts was retrieved from UCSC genome database (mm9). The same $z$ score \pm 1.5 cutoff was used. Group sizes: GS TG (TE up: 687, TE down: 335), KO (TE up: 596 , TE down: 576). Statistical significance was determined by one-way ANOVA with Bonferroni correction. $\boldsymbol{C}, \boldsymbol{D}$, Genes with complex 5'UTR secondary structure (estimated folding energy: $<-250 \mathrm{kcal} / \mathrm{mol}, 1145$ genes) or simple 5'UTR secondary structure (>-20 $\mathrm{kcal} / \mathrm{mol}, 1036$ genes) were selected, and the TE differences between $(\boldsymbol{C})$ : GS LRRK2 TG mice and control mice (D): LRRK2 KO mice and WT mice were plotted. Statistical significance was tested with Wilcoxon signed-rank test [C: $p<0.001$ (simple), $p=0.03533$ (complex); $\boldsymbol{D}: p=0.6007$ (simple), $p<0.001$ (complex)]. 3'UTR structures do not show correlation (Extended Data Fig. 2-1). $\boldsymbol{E}, \boldsymbol{F}$, Differential icSHAPE reactivity profiles between TE up and TE down genes. The same TE up and TE down genes with $z$ score \pm 1.5 were used; (E) GS TG (TE up: 687, TE down: 335), (F) LRRK2 KO (TE up: 596, TE down: 576). icSHAPE data from mouse ES cells were extracted (Spitale et al., 2015), and a window of -100-0 nt 5' of start codon (CDS start) was used. Average icSHAPE reactivity values: all genes: 0.236, TE up (GS): 0.229, TE down (GS): 0.240, TE up (KO): 0.237, TE down (KO): 0.219. Statistical significance (compared with all genes) was measured by non-parametric Mann-Whitney test. Error bars indicate SEM, ${ }^{*} p<0.05$, ${ }^{* *} p<0.01,{ }^{* * *} p<0.001$.

While we sought to characterize translational abnormalities in the LRRK2 KO mouse brain, we found unexpected patterns of ribosomal footprint distribution on the Atf4 upstream open reading frame (ORF) regions. Atf4 is the key transcription factor underlying one branch of the integrated stress response (ISR) pathway and its expression is known to be translationally regulated (Vattem and Wek, 2004). elF2a-mediated regulation of Atf4 is a well-studied example of translational regulation using termination-reinitiation balance between the upstream ORFs. We observed that in the LRRK2 KO brain, ribosome footprints are depleted 15-20 nt before the start codon of the main ORF (Fig. 4A). We performed additional ribosome profiling experiments with the caudate putamen (striatum; STR) and the ventral midbrain (VMB) of LRRK2 KO mouse brain and found that the footprint depletion is consistent across all ribosome profiling experiments conducted (Fig. 4B). Since we found that 5'UTR secondary structure is important to LRRK2-mediated translational regulation, 
A

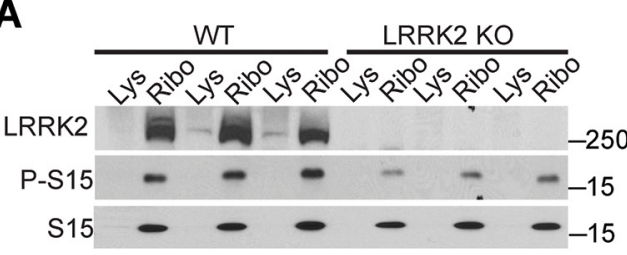

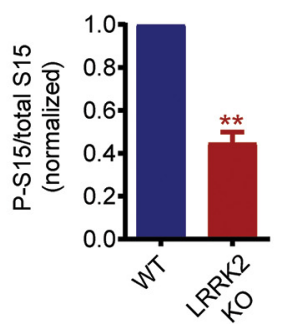

B

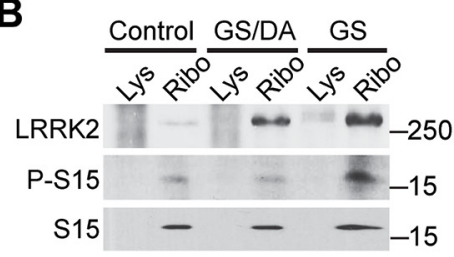

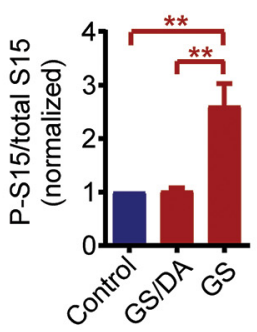

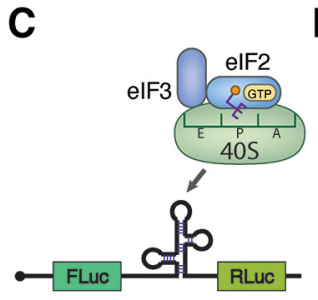

HCV-IRES
E

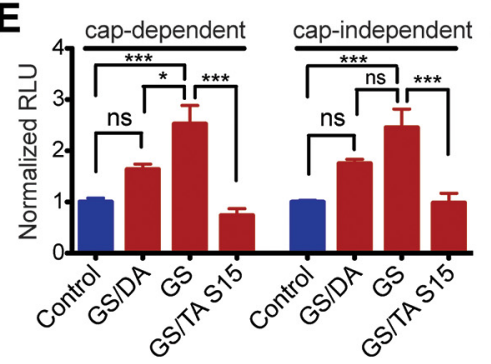

H

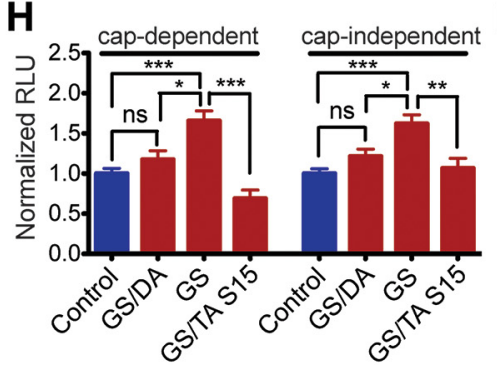

$F$

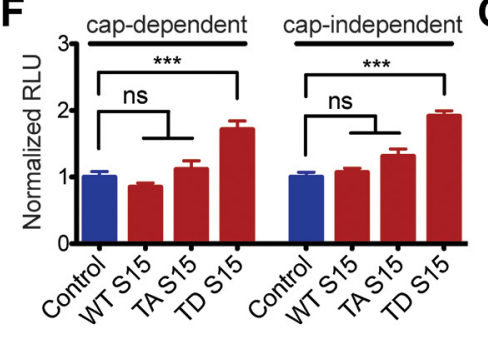

G

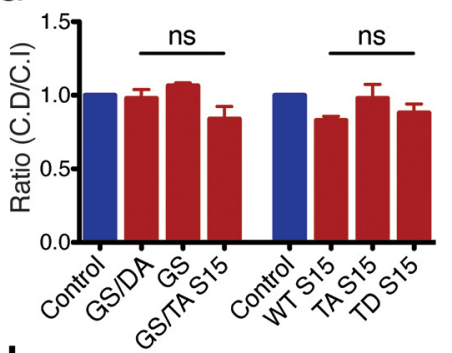

$\mathrm{J}$
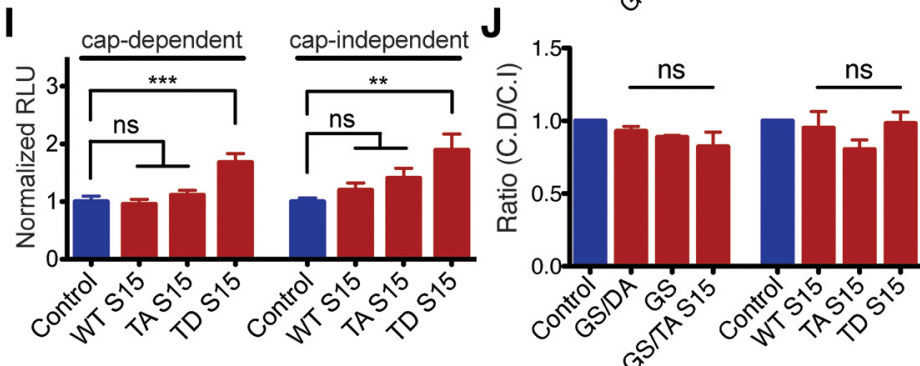

Figure 3. G2019S LRRK2 increases mRNA translation independent of initiation factors. $\boldsymbol{A}, \boldsymbol{B}$, Western blotting and quantification of T136 S15 phosphorylation in the mouse brain. LRRK2 KO $(\boldsymbol{A})$ and G2019S LRRK2 transgenic $(\boldsymbol{B})$ mice. Whole-brain lysate was used. $n=3$, biological replicates. Statistical significance was determined by $(\boldsymbol{A})$ unpaired $t$ test $(\boldsymbol{B})$ one-way ANOVA with Bonferroni correction. $\boldsymbol{C}, \boldsymbol{D}$, Schematics of HCV-IRES and CrPV-IRES reporters. $\boldsymbol{E}-\boldsymbol{G}$, HCV-IRES reporter assays. $\boldsymbol{C}, n=4 ; \boldsymbol{D}, n=3$, respectively. $\boldsymbol{H}-\boldsymbol{J}$, CrPV IRES reporter assays. $\boldsymbol{F}, n=4 ; \boldsymbol{G}, n=3$, respectively. Reporter assays were performed in primary mouse cortical neurons with transient transfection, and each experiment is an average of triplicates. All values were divided by the average of control values. Reporter mRNA levels were controlled (Extended Data Fig. 3-1). WT, wild type; Fluc, firefly luciferase; RLuc, Renilla luciferase; RLU, relative light units. Statistical significance was determined by one-way ANOVA with Bonferroni correction. Error bars indicate SEM, ${ }^{*} p<0.05,{ }^{* *} p<0.01,{ }^{* * *} p<0.001$, ns $=$ no significance.

we examined potential secondary structures near the depleted region. Computational secondary structure predictions (RNAfold) reported multiple potential hairpins in the Atf4 mRNA, and the depleted region in particular has a very high probability to form hairpin (Fig. 4C). These results further point to the importance of $5^{\prime}$ UTR secondary structure near start codon for the translational effects of LRRK2.

Since Atf4 uORF footprint abnormality is observed in the LRRK2 KO brain and Atf4 induction is a central downstream pathway of ISR, we further sought to determine a potential relationship between G2019S LRRK2 and ISR. First, elF2a phosphorylation levels in the G2019S LRRK2 transgenic brain were examined. We found no steadystate induction of elF2a phosphorylation regardless of the transgene expression levels (Extended Data Fig. 4$1 A)$. Next, we tested the potential relationship by inducing ISR in G2019S LRRK2-expressing primary neurons cultured from the transgenic mouse model. Of note, G2019S LRRK2-expressing neurons have defective recovery from thapsigargin-induced ISR (Extended Data Fig. 4-1B). Considering the enhanced translation of structured
5'UTR-containing transcripts in the G2019S LRRK2 brain, this defected recovery could be because of translational defects caused by G2019S LRRK2 inhibiting 5'UTR-mediated translational regulation required for ISR recovery. In addition, since thapsigargin induces ISR by blocking SERCA, defective calcium handling in G2019S LRRK2 neurons could also exacerbate ISR. A previous study suggested that dysregulated translation leads to increased calcium influx in G2019S LRRK2 human dopamine neurons (Kim et al., 2020). In this regard, we performed calcium recordings with the G2019S LRRK2-expressing brain. Basic electrophysiological properties including spontaneous and evoked AP wave of SNpc dopamine neurons are indifferent to G2019S LRRK2 expression in the brain (Extended Data Fig. 5-1). Substantia nigra dopamine neurons also show similar pacemaking activities compared with the wild type (Fig. 5A). However, calcium currents measurement showed significant increase of calcium currents in the G2019S LRRK2-expressing brain (Fig. 5B,C). These results are consistent with the previous report of increased calcium influx in G2019S LRRK2 human dopamine neurons (Kim et al., 2020). 
A

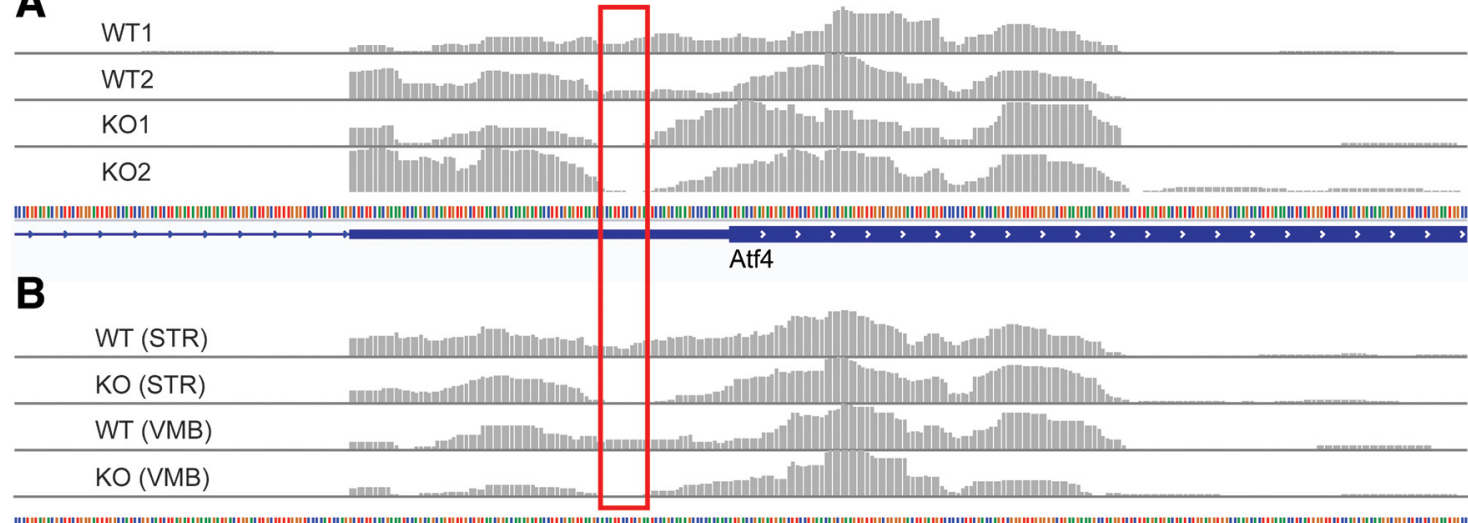

\section{C}

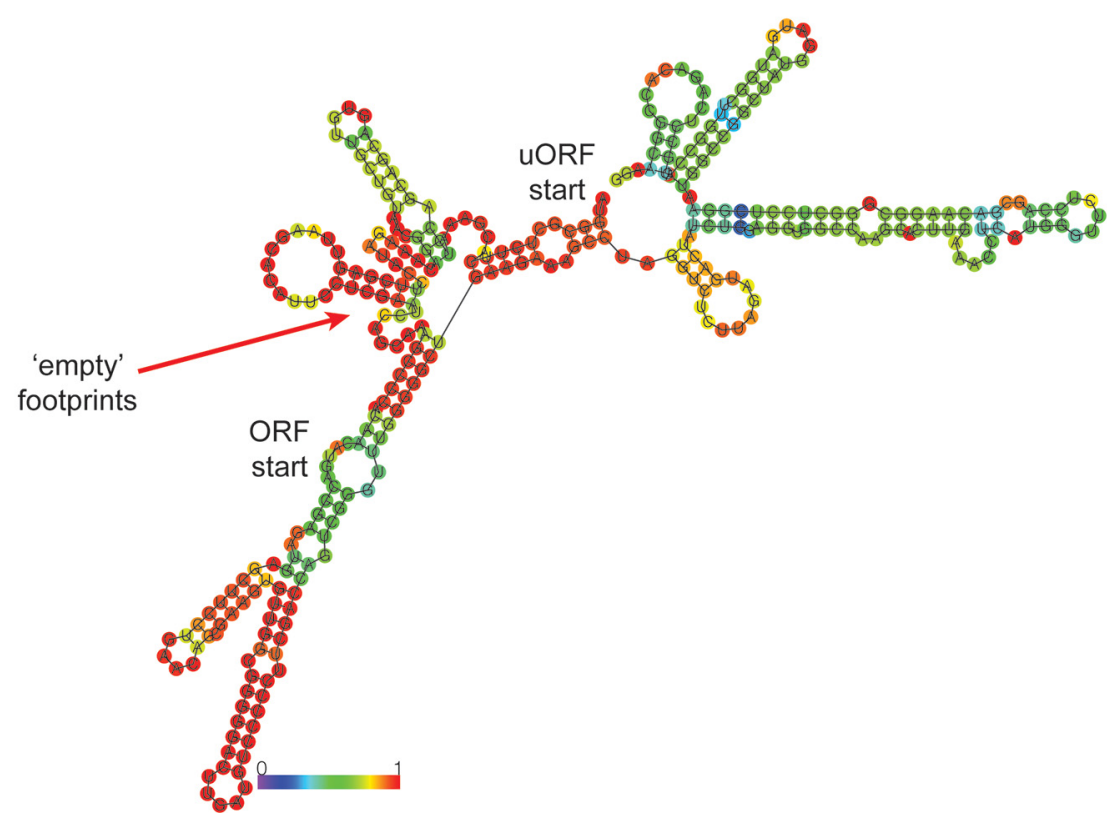

Figure 4. Ribosome footprint distributions on Atf4 uORFs in the LRRK2 KO brain. $\boldsymbol{A}, \boldsymbol{B}$, Ribosome footprints distribution in the 5'UTR of Atf4 gene (visualized: chr15:80,086,569-80,086,862). Red box indicates the region that ribosomes are depleted in the LRRK2 KO brain. $\boldsymbol{C}$, RNA structure prediction of the Atf4 uORF sequences by ViennaRNA RNAfold (Lorenz et al., 2011). The regions of depleted ribosome footprints have high probability to form secondary structure. In addition, relationship between G2019S LRRK2 and elF2a was addressed (Extended Data Fig. 4-1).

\section{Discussion}

In this study, we found that G2019S LRRK2 alters the global translational landscape in the mouse brain. Dysregulated translation caused by G2019S LRRK2 has been reported in Drosophila models and human dopamine neurons differentiated from patient-derived iPSCs (Martin et al., 2014b; Kim et al., 2020). Our data from the mouse brain are in line with the previous results showing that G2019S LRRK2 induces genome-wide translational abnormality. In addition, the 5'UTR-mediated translational shift, which was previously observed in the G2019S LRRK2 human dopamine neurons, is also present in the mouse brain. In G2019S LRRK2 transgenic mice, mRNAs with complex 5'UTR secondary structure tend to have elevated TE, while LRRK2 KO mice show the opposite trend. Analysis of RNA secondary structure data suggest that mRNA secondary structure on the 5'UTR regions near the start codon is important for these TE changes. These observations are in accordance with the previous finding that G2019S LRRK2 alters genome-wide translation by favoring mRNAs with complex 5'UTR secondary structure.

While the precise structural mechanism underlying the 5'UTR-mediated mRNA preference is unclear, our IRES reporter assays suggest that the enhanced translation in G2019S LRRK2 expressing neurons does not rely on 
A

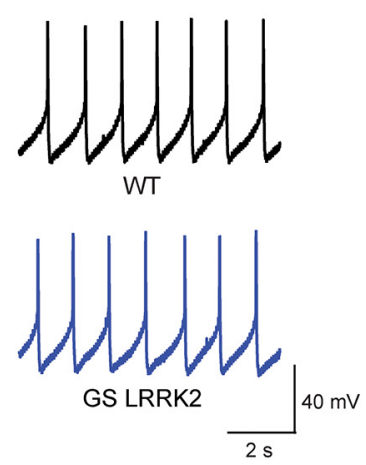

B

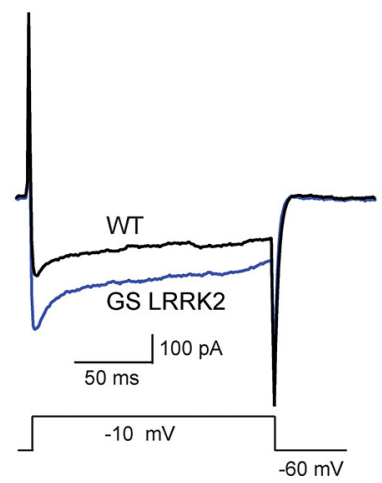

C

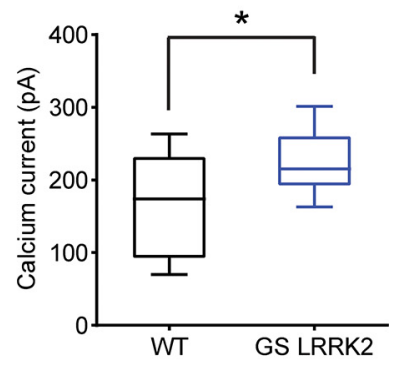

Figure 5. Calcium currents recorded in SNpc DA neurons. A, Comparison of spontaneous AP firing pattern of DA neurons between wild-type and GS LRRK2 mouse slices. $\boldsymbol{B}$, Calcium currents were measured in mouse SNpc DA neurons using whole-cell patch clamp recordings. $\boldsymbol{C}$, Quantification of calcium peak currents. Data are expressed as means \pm SEM, $n=12$ slices from 12 animals for each group. Intrinsic properties were measured (Extended Data Fig. 5-1).

translation initiation factors. Considering that G2019S LRRK2 is known to phosphorylate multiple ribosomal proteins including S15, our results bolster the idea that phosphorylation of ribosomal proteins could change the global translational landscape autonomously. Of note, the IRES reporter assays also indicate that the effects of LRRK2 may not be limited to translational initiation, since IRESrecruited ribosomes are thought to bypass scanning (Jackson et al., 2010). While our analyses indicate a strong correlation between $5^{\prime}$ UTR secondary structure and TE, an alteration in secondary structure may in theory impact elongation as well. Secondary structure-mediated regulation is generally considered in the context of translation initiation since the coding region has limited degree of freedom for nucleotide-based secondary structure formation. However, as the Atf4 CDS secondary structure prediction depicts, it is possible that elongation could be, at least partially, regulated by mRNA secondary structure as well. Therefore, these collectively suggest that the G2019S LRRK2 mutation and its downstream effects can facilitate translation during both initiation and elongation steps if secondary structure-mediated regulation is in place.

Ribosome footprint depletion at the Atf4 $5^{\prime}$ UTR in LRRK2 KO provides new information on the mechanisms by which LRRK2 affects translation. It suggests that the low TE of complex $5^{\prime}$ UTR genes in the LRRK2 KO brain is because of strong hairpin formation and reduced ribosomal processivity. Alternatively, it is possible that the depletion is caused by disome formation, which can reduce the recovery of footprints in ribosome profiling experiments that include a monosome-specific size-selection step. This disome hypothesis is supported by the facts that the depletion is just in front of the main CDS start codon, and the main CDS also tends to form strong hairpin structure right after the start codon (Fig. 4C). Both cases are consistent with the interpretation of reduced ribosomal processivity in the LRRK2 KO brain. It further suggests that uORF-mediated regulation of Atf4 expression could potentially be regulated by manipulating ribosomal processivity. While we did not find any Atf4 footprint distribution abnormality in the G2019S LRRK2 transgenic brain, we cannot exclude the possibility that increased ribosomal processivity could impair the ISR, thereby incurring a long-term cellular stress in G2019S LRRK2 PD. Delayed recovery from thapsigargin-mediated ISR in G2019S LRRK2 neurons might be linked to this increased processivity. Since ATF4 plays central roles in the integrated stress responses, including induction of genes necessary to cope with cellular stresses, understanding the exact molecular mechanisms for Atf4 expression regulation will deepen our knowledge on the pathobiology of LRRK2 PD.

Since this study was conducted with dissected brain tissues without cell-type specificity, dopamine neuronspecific translational profiling experiments in rodent models, which have been done in human iPSC-derived dopamine neuron and Drosophila models, would further reveal the specific changes relevant to G2019S LRRK2 PD (Kim et al., 2020; Pallos et al., 2021). In addition, there is a recent report suggesting that G2019S LRRK2 leads to reduced bulk translation in rodent neurons (Deshpande et al., 2020). The study was conducted with different model systems from this study (cultured neurons, in vitro translation system, and skin fibroblasts), which makes it hard to directly compare the results. However, bulk protein synthesis rate is tightly related to the neuronal activity levels. In this regard, investigating the relationship between LRRK2 and neuronal activity would be informative to collectively comprehend the molecular mechanisms of LRRK2-mediated translational regulation.

It is noteworthy that calcium influx is increased in the substantia nigra dopamine neurons in the G2019S LRRK2-expressing brain. The increased calcium influx was originally reported in G2019S LRRK2 human dopamine neurons. While the previous findings from cultured neurons initiated a plausible molecular mechanism that can led to a long-term dopamine neuronal stress, the electrophysiological characteristic of a neuron is heavily influenced by the neuron's wiring context. Therefore, monitoring calcium dynamics in a fully developed adult brain tissue is essential to validate the hypothesis (Yin et al., 2021). In this manner, our findings on the increased calcium influx in vivo bolster the suggested molecular 
etiology that calcium dysregulation leads to dopamine neuronal stress in the G2019S LRRK2 PD.

\section{References}

Anders S, Huber W (2010) Differential expression analysis for sequence count data. Genome Biol 11:R106.

Andres-Mateos E, Mejias R, Sasaki M, Li X, Lin BM, Biskup S, Zhang L, Banerjee R, Thomas B, Yang L, Liu G, Beal MF, Huso DL, Dawson TM, Dawson VL (2009) Unexpected lack of hypersensitivity in LRRK2 knock-out mice to MPTP (1-methyl-4-phenyl-1,2,3,6tetrahydropyridine). J Neurosci 29:15846-15850.

Brar GA, Weissman JS (2015) Ribosome profiling reveals the what, when, where and how of protein synthesis. Nat Rev Mol Cell Biol 16:651-664.

Deshpande P, Flinkman D, Hong Y, Goltseva E, Siino V, Sun L, Peltonen S, Elo LL, Kaasinen V, James P, Coffey ET (2020) Protein synthesis is suppressed in sporadic and familial Parkinson's disease by LRRK2. FASEB J 34:14217-14233.

Gehrke S, Imai Y, Sokol N, Lu B (2010) Pathogenic LRRK2 negatively regulates microRNA-mediated translational repression. Nature 466:637-641.

Greggio E, Jain S, Kingsbury A, Bandopadhyay R, Lewis $P$, Kaganovich A, van der Brug MP, Beilina A, Blackinton J, Thomas KJ, Ahmad R, Miller DW, Kesavapany S, Singleton A, Lees A, Harvey RJ, Harvey K, Cookson MR (2006) Kinase activity is required for the toxic effects of mutant LRRK2/dardarin. Neurobiol Dis 23:329-341.

Imai Y, Gehrke S, Wang HQ, Takahashi R, Hasegawa K, Oota E, Lu B (2008) Phosphorylation of 4E-BP by LRRK2 affects the maintenance of dopaminergic neurons in Drosophila. EMBO J 27:24322443.

Ingolia NT (2016) Ribosome footprint profiling of translation throughout the genome. Cell 165:22-33.

Ingolia NT, Ghaemmaghami S, Newman JR, Weissman JS (2009) Genome-wide analysis in vivo of translation with nucleotide resolution using ribosome profiling. Science 324:218-223.

Ingolia NT, Brar GA, Rouskin S, McGeachy AM, Weissman JS (2012) The ribosome profiling strategy for monitoring translation in vivo by deep sequencing of ribosome-protected mRNA fragments. Nat Protoc 7:1534-1550.

Jackson RJ, Hellen CU, Pestova TV (2010) The mechanism of eukaryotic translation initiation and principles of its regulation. Nat Rev Mol Cell Biol 11:113-127.

Kim JW, Yin X, Jhaldiyal A, Khan MR, Martin I, Xie Z, Perez-Rosello $\mathrm{T}$, Kumar M, Abalde-Atristain L, Xu J, Chen L, Eacker SM, Surmeier DJ, Ingolia NT, Dawson TM, Dawson VL (2020) Defects in mRNA translation in LRRK2-mutant hiPSC-derived dopaminergic neurons lead to dysregulated calcium homeostasis. Cell Stem Cell 27:633-645.e7.

Lee Y, Karuppagounder SS, Shin JH, Lee YI, Ko HS, Swing D, Jiang H, Kang SU, Lee BD, Kang HC, Kim D, Tessarollo L, Dawson VL, Dawson TM (2013) Parthanatos mediates AIMP2-activated agedependent dopaminergic neuronal loss. Nat Neurosci 16:13921400.
Lorenz R, Bernhart SH, Honer Zu Siederdissen C, Tafer H, Flamm C, Stadler PF, Hofacker IL (2011) ViennaRNA package 2.0. Algorithms Mol Biol 6:26.

Martin I, Kim JW, Dawson VL, Dawson TM (2014a) LRRK2 pathobiology in Parkinson's disease. J Neurochem 131:554-565.

Martin I, Kim JW, Lee BD, Kang HC, Xu JC, Jia H, Stankowski J, Kim MS, Zhong J, Kumar M, Andrabi SA, Xiong Y, Dickson DW, Wszolek ZK, Pandey A, Dawson TM, Dawson VL (2014b) Ribosomal protein $\mathrm{s} 15$ phosphorylation mediates LRRK2 neurodegeneration in Parkinson's disease. Cell 157:472-485.

Nikonova EV, Xiong Y, Tanis KQ, Dawson VL, Vogel RL, Finney EM, Stone DJ, Reynolds IJ, Kern JT, Dawson TM (2012) Transcriptional responses to loss or gain of function of the leucine-rich repeat kinase 2 (LRRK2) gene uncover biological processes modulated by LRRK2 activity. Hum Mol Genet 21:163-174.

Pallos J, Jeng S, McWeeney S, Martin I (2021) Dopamine neuronspecific LRRK2 G2019S effects on gene expression revealed by translatome profiling. Neurobiol Dis 155:105390.

Parsyan A, Svitkin Y, Shahbazian D, Gkogkas C, Lasko P, Merrick WC, Sonenberg N (2011) mRNA helicases: the tacticians of translational control. Nat Rev Mol Cell Biol 12:235-245.

Sen ND, Zhou F, Ingolia NT, Hinnebusch AG (2015) Genome-wide analysis of translational efficiency reveals distinct but overlapping functions of yeast DEAD-box RNA helicases Ded1 and elF4A. Genome Res 25:1196-1205.

Smith WW, Pei Z, Jiang H, Dawson VL, Dawson TM, Ross CA (2006) Kinase activity of mutant LRRK2 mediates neuronal toxicity. Nat Neurosci 9:1231-1233.

Sonenberg N, Hinnebusch AG (2009) Regulation of translation initiation in eukaryotes: mechanisms and biological targets. Cell 136:731-745.

Spitale RC, Flynn RA, Zhang QC, Crisalli P, Lee B, Jung JW, Kuchelmeister HY, Batista PJ, Torre EA, Kool ET, Chang HY (2015) Structural imprints in vivo decode RNA regulatory mechanisms. Nature 519:486-490.

Taymans JM, Nkiliza A, Chartier-Harlin MC (2015) Deregulation of protein translation control, a potential game-changing hypothesis for Parkinson's disease pathogenesis. Trends Mol Med 21:466472.

Thoreen CC, Chantranupong L, Keys HR, Wang T, Gray NS, Sabatini DM (2012) A unifying model for mTORC1-mediated regulation of mRNA translation. Nature 485:109-113.

Vattem KM, Wek RC (2004) Reinitiation involving upstream ORFs regulates ATF4 mRNA translation in mammalian cells. Proc Natl Acad Sci USA 101:11269-11274.

Xiong Y, Neifert S, Karuppagounder SS, Stankowski JN, Lee BD, Grima JC, Chen G, Ko HS, Lee Y, Swing D, Tessarollo L, Dawson TM, Dawson VL (2017) Overexpression of Parkinson's disease-associated mutation LRRK2 G2019S in mouse forebrain induces behavioral deficits and $\alpha$-synuclein pathology. eNeuro 4:ENEURO.0004-17.2017.

Yin X, Kim JW, Liu S, Dawson TM, Dawson VL (2021) Protocol for measurement of calcium dysregulation in human induced pluripotent stem cell-derived dopaminergic neurons. STAR Protoc 2:100405 